\title{
Combined Inhibitor Free-Energy Landscape and Structural Analysis Reports on the Mannosidase Conformational Coordinate**
}

\author{
Rohan J. Williams, Javier Iglesias-Fernández, Judith Stepper, Adam Jackson, \\ Andrew J. Thompson, Elisabeth C. Lowe, Jonathan M. White, Harry J. Gilbert, Carme Rovira,* \\ Gideon J. Davies, * and Spencer J. Williams*
}

\begin{abstract}
Mannosidases catalyze the hydrolysis of a diverse range of polysaccharides and glycoconjugates, and the various sequence-based mannosidase families have evolved ingenious strategies to overcome the stereoelectronic challenges of mannoside chemistry. Using a combination of computational chemistry, inhibitor design and synthesis, and X-ray crystallography of inhibitor/enzyme complexes, it is demonstrated that mannoimidazole-type inhibitors are energetically poised to report faithfully on mannosidase transition-state conformation, and provide direct evidence for the conformational itinerary used by diverse mannosidases, including $\beta$-mannanases from families GH26 and GH113. Isofagomine-type inhibitors are poor mimics of transition-state conformation, owing to the high energy barriers that must be crossed to attain mechanistically relevant conformations, however, these sugarshaped heterocycles allow the acquisition of ternary complexes that span the active site, thus providing valuable insight into active-site residues involved in substrate recognition.
\end{abstract}

$M$ annosidases are glycoside hydrolases (GHs) which catalyze the cleavage of glycosidic linkages in mannose-containing glycoconjugates and polysaccharides. $\alpha$-Mannosidases are important in $\mathrm{N}$-glycan biosynthesis and protein quality control and their inhibition may allow intervention in diseases which utilize $\mathrm{N}$-linked glycans for protein folding. ${ }^{[1]} \beta$ Mannosidases are important in the degradation of plantderived mannans ( $\beta$-mannan, glucomannan, galactomannan) and are of industrial significance in the detergent, food, biofuels, and oil and gas industries. ${ }^{[2]}$ Biochemical studies of mannosidases from different sequence-based families have highlighted that a variety of conformational itineraries, ${ }^{[3]}$ and a range of mechanistic strategies are employed for glycosidic bond cleavage. ${ }^{[4]}$ The study of the diverse pathways employed by mannosidases can inform synthetic efforts designed to overcome the often recalcitrant chemistry of mannose. ${ }^{[5]}$

A rationalization of the rate enhancement achieved by an enzyme pivots upon an understanding of its transition state. This understanding in turn can instruct the development of transition-state analogue inhibitors, which have exciting potential as drug candidates. ${ }^{[6]}$ Mannosidase inhibitors are designed to mimic the charge, planarity, and conformation of the oxocarbenium ion-like "exploded" transition state(s) of mannosidase-catalyzed hydrolysis. Upon protonation, isofagomine-type inhibitors, exemplified by isofagomine (IFG) 1 (Figure 1), resemble a glycosyl cation with charge localized at $\mathrm{C} 1$, but are poor mimics of transition-state conformation.

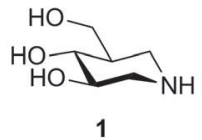

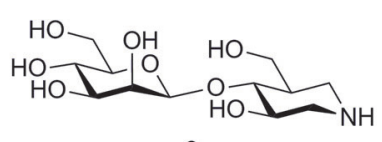

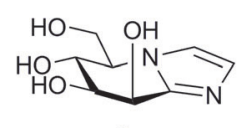

2

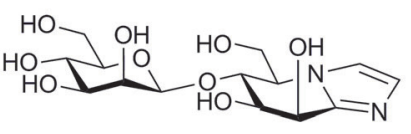

Figure 1. Structures of isofagomine (1), mannoimidazole (2), ManIFC (3), and ManMIm (4).
[*] Dr. R. J. Williams, ${ }^{[+]}$Prof. J. M. White, Prof. S. J. Williams

School of Chemistry and Bio21 Molecular Science and

Biotechnology Institute, University of Melbourne

Parkville, Vic 3010 (Australia)

E-mail: sjwill@unimelb.edu.au

J. Iglesias-Fernández, ${ }^{[+]}$Prof. C. Rovira

Departament de Química Orgànica and Institut de Química Teòrica

i Computacional (IQTCUB), Universitat de Barcelona

08028 Barcelona (Spain)

and

Institut de Química Teòrica i Computacional (Spain)

E-mail: crovira@pcb.ub.es

Prof. C. Rovira

Institució Catalana de Recerca i Estudis Avançats (ICREA)

Passeig Lluís Companys 23, 08020 Barcelona (Spain)

Dr. J. Stepper, ${ }^{[+]}$Dr. A. J. Thompson, Prof. G. J. Davies

Department of Chemistry, University of York

Heslington, York, YO10 5DD (UK)

E-mail: gideon.davies@york.ac.uk
A. Jackson, Dr. E. C. Lowe, Prof. H. J. Gilbert

Institute for Cell and Molecular Biosciences, The Medical School

Newcastle University, Newcastle upon Tyne, NE2 4HH (UK)

$\left.{ }^{+}\right]$These authors contributed equally to this work.

$[* *$ We thank the Australian Research Council, the UK Biotechnology and Biological Sciences Research Council, the Spanish Ministry of Economy and Competitiveness, the Generalitat de Catalunya, the Albert Shimmins bequest, and Amicus Therapeutics. We acknowledge the support, technical expertise, and assistance provided by the Barcelona Supercomputing Center: Centro Nacional de Supercomputación.

Supporting information for this article is available on the WWW under http://dx.doi.org/10.1002/anie.201308334.

of (c) 2014 The Authors. Published by Wiley-VCH Verlag GmbH \& Co. $\mathrm{KGaA}$. This is an open access article under the terms of the Creative Commons Attribution License, which permits use, distribution and reproduction in any medium, provided the original work is properly cited. 
Alternatively, mannoimidazole (MIm)-type inhibitors, for example, $\mathbf{2}$, are qualitatively good models of a mannopyranosyl oxocarbenium ionlike transition state, with $\mathrm{sp}^{2}$ hybridization of equivalent atoms and the potential for anti protona$\operatorname{tion}^{[7]}$ of the imidazole functionality. Structural studies aimed at identifying the conformation of the transition state and flanking ground states on the reaction coordinate, utilizing $\mathbf{2}$, and related inhibitors as transitionstate and ground-state mimics, identified a ${ }^{1} S_{5} \leftrightarrow B_{2,5}{ }^{\neq} \leftrightarrow{ }^{\mathrm{O}} S_{2}$ conformational itinerary for mannosidases of families $\mathrm{GH} 2{ }^{[8]} 38,{ }^{[9]}$ and $92,{ }^{[10]}$ and a ${ }^{3} S_{1} \rightarrow{ }^{3} H_{4}{ }^{\neq} \rightarrow{ }^{1} C_{4}$ itinerary for the $\alpha$ mannosidases of GH47. ${ }^{[11]}$ However, it is now apparent that complexes with 2 can report on both the transition-state conformation and conformational itinerary. ${ }^{[11]}$ Quantitative kinetic and thermodynamic analyses using Bartlett plots and isothermal titration calorimetry suggest $\mathbf{2}$ is a true transition-state mimic. ${ }^{[8,11]}$ However, these energetic analyses do not report which features of the ligand imbue transition-state mimicry.

Herein we present syntheses of new $\beta$-mannanase-targeted inhibitors, ManIFG (3) and ManMIm (4; Figure 1). Together with 1,4- $\beta$ mannobiose, $\mathbf{3}$ afforded a unique ternary complex with the $\beta$ mannanase of family GH113, thus defining the active-site cleft. Compound 4 was used to obtain new transition-state mimicking complexes with $\beta$-mannanases of families GH26 and GH113, thus allowing the assignment of conformational itineraries for these enzymes. The conformations of the inhibitors in these novel structures and previously reported mannosidase complexes of these classes of inhibitors were correlated with quantum mechanical (QM) calculations of the free-energy landscape (FEL) for $\mathbf{1}$ and $\mathbf{2}$ "off-enzyme", to identify which conformations better resemble the mannosidase transition state.

A ${ }^{1} S_{5} \rightarrow B_{2,5}{ }^{\neq} \rightarrow{ }^{\mathrm{O}} S_{2}$ glycosylation conformational itinerary has been proposed for GH26 mannanases on the basis of the transition-state flanking Michaelis complex in a ${ }^{1} S_{5}$ conformation, ${ }^{[12]}$ and the glycosyl-enzyme intermediate in an ${ }^{\circ} S_{2}$ conformation, ${ }^{[13]}$ combined with the principle of least nuclear motion. Only the uncomplexed form of a sole GH113 $\beta$ mannanase has been solved and no proposals have been advanced for transition-state conformation for this family. ${ }^{[14]}$ To gain direct evidence for transition-state conformation for these two families, the isofagomine and mannoimidazole inhibitor "warheads" were used to develop new inhibitors targeted at the representative $\beta$-mannanases from families GH26 and GH113, $\beta$-1,4-mannobiohydrolase from Cellvibrio japonicus CjMan26C, and endo- $\beta-1,4-m a n n a n a s e$ from Alicyclobacillus acidocaldarius AaManA, respectively. The synthesis of $\mathbf{3}$ and $\mathbf{4}$ is outlined in Scheme 1 (see the Supporting Information).

Compounds $\mathbf{3}$ and $\mathbf{4}$ are potent inhibitors of CjMan26C with $K_{\mathrm{I}}$ values of $(263 \pm 15)$ and $(194 \pm 8) \mathrm{nM}$, respectively, thus partly reflecting an unusually high-affinity -2 subsite. ${ }^{[15]}$ Soaking $\mathbf{3}$ and $\mathbf{4}$ into crystals of $C j \mathrm{Man} 26 \mathrm{C}$ yielded complexes that diffracted to a resolution of 1.2 and $1.1 \AA$, respectively, with inhibitors occupying the -2 and -1 subsites (Figure $2 \mathrm{a}$; see Figures S1a, S2; Table S1 in the Supporting Information). The MIm moiety in the -1 subsite of the $C j \mathrm{Man} 26 \mathrm{C} / 4$ complex adopts a $B_{2,5}$ conformation, which is consistent with the proposed ${ }^{1} S_{5} \rightarrow B_{2,5}{ }^{\neq} \rightarrow{ }^{\mathrm{O}} S_{2}$ glycosylation conformational itinerary for $\mathrm{GH} 26$.

Compounds 3 and $\mathbf{4}$ are weaker inhibitors of $A a \mathrm{ManA}$ with $K_{\mathrm{I}}$ values of $(0.26 \pm 0.04)$ and $1.3 \mathrm{~mm}$, respectively, which are consistent with this enzyme exhibiting a preference for binding elongated substrates in the - and + subsites distal to -2 and -1 . Soaking 4 into crystals of $A a$ ManA yielded a complex that diffracted to a resolution of $1.5 \AA$ with the ligand occupying the -2 and -1 subsites. The MIm moiety in the -1 subsite adopts a $B_{2,5}$ conformation (Figure $2 \mathrm{~b}$; see Figure S2 and Table S1). By invoking the principle of least nuclear motion, this complex is diagnostic of a ${ }^{1} S_{5} \rightarrow B_{2,5}{ }^{\neq} \rightarrow{ }^{\mathrm{O}} S_{2}$ conformational itinerary for family GH113. Soaking $\mathbf{3}$, either 
a)

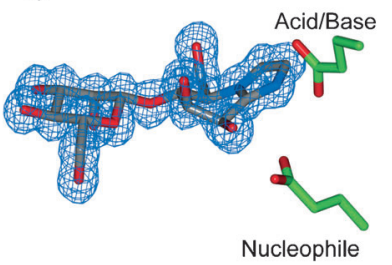

c)

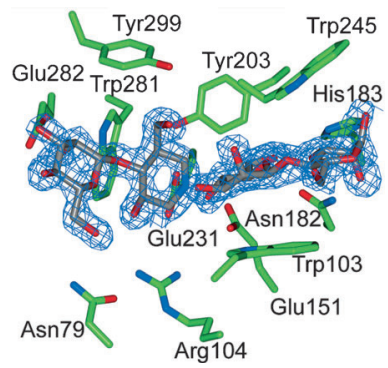

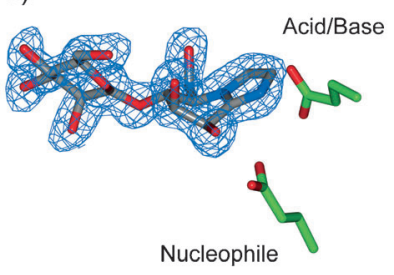

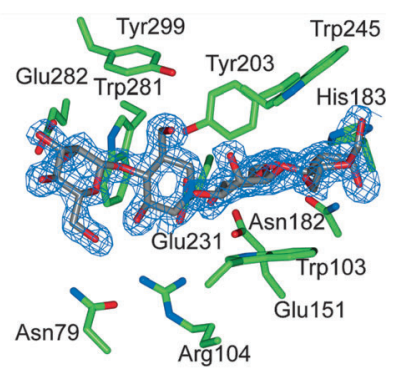

Figure 2. a) Binary complex of ManMIm (4) bound to CjMan26C. b) Binary complex of $\mathbf{4}$ bound to AaManA. c) Ternary complex showing ligand binding within the active site of $\mathrm{GH} 113 \beta$-mannanase AaManA. ManIFC (3) occupies the -2 and -1 subsites, whilst $\beta$-1,4-mannobiose is observed within +1 and +2 subsites. Depicted electron density maps are REFMAC maximum-likelihood $/ \sigma_{A}$-weighted $2 F_{o}-F_{c}$ syntheses contoured at $0.41,0.38$, and 0.41 electrons per $\AA^{3}$, respectively.

alone, or with $1,4-\beta$-mannobiose, into crystals of $A a$ ManA yielded binary and ternary complexes which diffracted to a resolution of $1.6 \AA$ (Figure 2c; see Figures S1b and S2, and Table S1). The ternary complex with $\mathbf{3}$ bound in the -2 and -1 subsites, and 1,4- $\beta$-mannobiose bound in the +1 and +2 subsites, provides the first complete mapping of substratebinding amino acid residues across the -2 to +2 subsites for a GH113 enzyme (Figure 2c; see Figure S2 and Table S1).

To understand the intrinsic conformational preferences of $\mathbf{1}^{[16]}$ and 2, we employed QM calculations to construct a conformational FEL. The FEL of 1 reveals that the ${ }^{4} C_{1}$ conformation is preferred, with the mechanistically relevant ${ }^{1} C_{4}$ and $B_{2,5}$ conformations located 5 and $8 \mathrm{kcal} \mathrm{mol}^{-1}$ higher in energy, respectively, and with a greater than $10 \mathrm{kcalmol}^{-1}$ barrier for their interconversion (Figure $3 \mathrm{a}$ ). These data suggest that IFG is a poor transition-state mimic and consistent with this the ${ }^{4} C_{1}$ conformation is the only conformation observed for isofagomine-type inhibitors when bound to mannosidases (Figure $3 \mathrm{a}$ ). Other conformations have been observed for isofagomine-type inhibitors bound to glucosidases/cellulases (see Figure S3). In striking contrast, the FEL of $\mathbf{2}$ is consistent with good transition-state shape mimicry, with all mechanistically relevant half-chair $\left({ }^{4} \mathrm{H}_{3}\right.$ and $\left.{ }^{3} H_{4}\right)$, envelope $\left({ }^{3} E, E_{3},{ }^{4} E\right.$, and $\left.E_{4}\right)$, and boat $\left({ }^{2,5} B\right.$ or $\left.B_{2,5}\right)$ conformations energetically accessible (Figure $3 \mathrm{~b}$ ). A global minimum was found near the ${ }^{4} H_{3}$ conformation with a second local minimum approximately $1 \mathrm{kcal} \mathrm{mol}^{-1}$ higher in energy near the ${ }^{3} \mathrm{H}_{4}$ conformation, both of which have been observed on-enzyme. The other conformation of $\mathbf{2}$, which has been observed on-enzyme, the $B_{2,5}$, was near a saddle point between these local minima and was $5 \mathrm{kcalmol}^{-1}$ higher in

a)
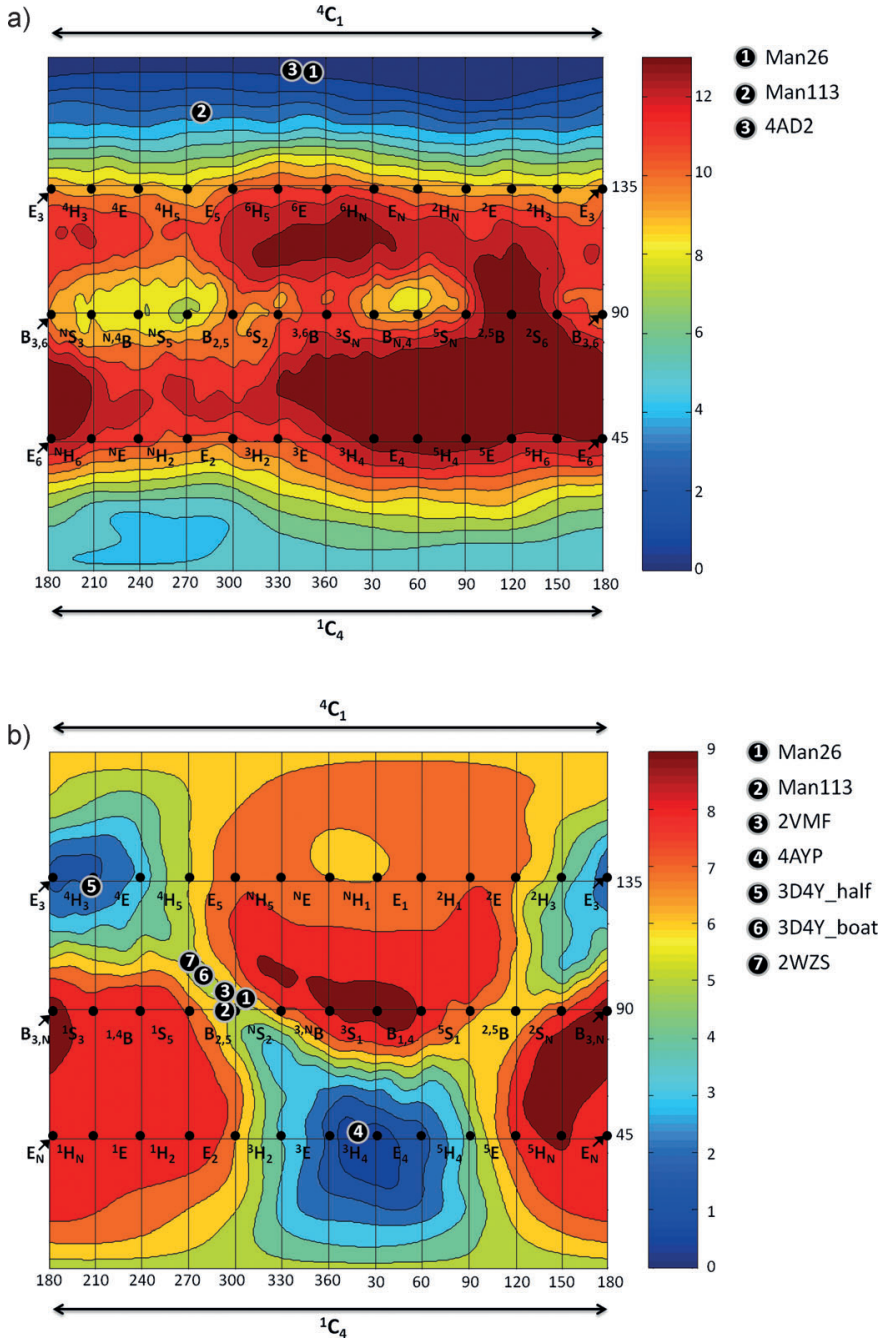

Figure 3. Conformational free-energy landscapes (FELs, Mercator projection) of isolated isofagomine $(\mathbf{1} ; \mathrm{a})$ and protonated mannoimidazole $(\mathbf{2}$; b), contoured at $1 \mathrm{kcal} \mathrm{mol}^{-1}$. FELs have been annotated with the conformations of isofagomine-type (for a) and mannoimidazole-type (for b) inhibitors which have been observed on-enzyme. a) 1: 3 bound to $\mathrm{GH} 26$ CjMan26C (this work); 2: 3 bound to $\mathrm{GH} 113$ AaManA (this work); 3: $\alpha$-Glc-1,3-isofagomine bound to BxGH99 (PDB code 4AD2). ${ }^{[1]}$ b) 1: 4 bound to $\mathrm{GH} 26$ CjMan26C (this work); 2: 4 bound to $\mathrm{GH} 113$ AaManA (this work); 3 : 1 bound to $\mathrm{GH} 2 \mathrm{BtMan2 \textrm {A }}$ (PDB code 2VMF) $;^{[18]} 4$ :

1 bound to GH47 CkMan47 (PDB code 4AYP) ; $;^{[1]} 5: 1$ bound to $\mathrm{GH} 38$ DmGManll (PDB code 3D4Y) in half-chair conformer; ${ }^{[19]} 6: 1$ bound to GH38 DmGManll (PDB code 3D4Y) in boat conformer (this work); 7: 1 bound to GH92 BtMan3990 (PDB code 2WZS). ${ }^{[2]}$

energy than the ${ }^{3} H_{4}$ conformation. The FEL for 2 reveals that the conformations relevant to the reaction coordinate are all energetically accessible, and moreover, that a $B_{2,5}$ conformation is less stable than the $E$ and $H$ conformations. This in turn suggests that the observation of a $B_{2,5}$ conformation for 2 onenzyme is of special mechanistic significance, with the enzyme inducing the inhibitor to adopt a conformation to match the transition state.

Collectively, the conformations of mannoimidazole-type inhibitors bound to mannosidases of diverse families highlights that mannosidases readily modulate the ligand conformational landscape. This conclusion is consistent with FEL 
analysis of $\alpha$-D-mannopyranose bound to a GH47 $\alpha$-mannosidase, and established that the enzyme reshapes the conformational landscape, thus defining the energetically accessible space. ${ }^{[11]}$ For enzymes from GH2, ${ }^{[21]} \mathrm{GH} 26,{ }^{[13]}$ and $\mathrm{GH} 47,{ }^{[11]}$ conformations of ground states adjacent to the transition state (Michaelis, glycosyl-enzyme or product complexes) provide strong evidence that mannoimidazole-type inhibitors authentically report transition-state conformation on-enzyme. However, the ${ }^{4} H_{3}$ conformation reported for the complex of 2 and Drosophila melanogaster Golgi GH38 $\alpha$ mannosidase II ( $D m$ GManII) is inconsistent with this interpretation. ${ }^{[19]}$ On the basis of a glycosyl-enzyme intermediate in a ${ }^{1} S_{5}$ conformation, ${ }^{[9]}$ and theory, ${ }^{[22]}$ a $B_{2,5}$ transition-state conformation is predicted. Inspection of the density map reveals significant residual electron density in the complex, ${ }^{[23]}$ and re-refinement of these data with $\mathbf{2}$ in two conformations shows that $30-40 \%$ of the ligand binds as a second transitionstate mimicking conformer in an approximate $B_{2,5}$ conformation (see Figure S4).

We next analyzed the atomic charges and ring planarity of the four mechanistically relevant conformations $\left({ }^{4} \mathrm{H}_{3},{ }^{3} \mathrm{H}_{4}\right.$, $B_{2,5},{ }^{2,5} B$ ) of 2 . We combined the values of the charge development at the $\mathrm{C} 1$ atom $\left(\mathrm{q}_{\mathrm{C} 1}\right)$, the $\mathrm{C} 5-\mathrm{O} 5-\mathrm{C} 1-\mathrm{C} 2$ dihedral angle, and the free energy for a large set of representative structures into a unique index (named "TS index", TSi, see the Supporting Information), in the spirit of the previously reported preactivation index for isolated aldohexoses. ${ }^{[1,24]}$ Table S2 and Figures S5 and S6 show that there is no single conformation with the optimum values for every parameter (score $=100$ ). The TSi values (see Figure S7) reveal that even though the $H$ conformations are favored over $B$ conformations when solely considering their energy (Figure $2 b$ ), they possess different TSi values. Most importantly, the two transition-state mimicking conformations that have been observed on-enzyme, ${ }^{3} \mathrm{H}_{4}$ and $B_{2,5}$, have the highest TSi values. Therefore, as observed for glycans bound to $\mathrm{GHs},{ }^{[3,25]}$ the inhibitor conformation on-enzyme is not only dictated by the relative energy of the molecule itself but also by structural and electronic properties.

In conclusion, the calculated FEL of $\mathbf{2}$ shows a preference for ${ }^{3} \mathrm{H}_{4}$ and ${ }^{4} \mathrm{H}_{3}$ half-chair conformations, and that a $B_{2,5}$ conformation represents a higher energy, but easily accessible saddle point between these two minima and thus that mannoimidazole-type inhibitors, in contrast to the isofagomine-type, are energetically poised to faithfully report transition-state conformation. X-ray structures of $\mathbf{4}$ with two $\beta$-mannanases from GH26 and GH113 provide the first direct
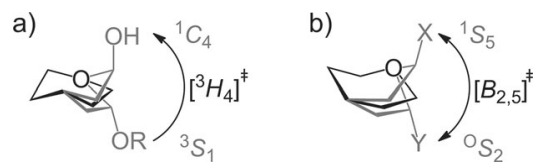

Figure 4. Conformational itineraries employed by mannosidases. a) ${ }^{3} \mathrm{~S}_{1} \rightarrow{ }^{3} \mathrm{H}_{4}{ }^{\neq} \rightarrow{ }^{1} \mathrm{C}_{4}$ itinerary of $\mathrm{GH} 47$ inverting $\alpha$-mannosidase. b) ${ }^{1} S_{5} \leftrightarrow B_{2,5}{ }^{\neq} \leftrightarrow{ }^{\circ} S_{2}$ itinerary employed by retaining $\mathrm{GH} 2$ and $\mathrm{GH} 38 \beta$ mannosidases, inverting $\mathrm{GH} 92 \alpha$-mannosidases and retaining $\mathrm{GH} 26$ and $\mathrm{GH} 113 \beta$-mannanases $(\mathrm{X}, \mathrm{Y}=$ leaving group, enzyme carboxylate or $\mathrm{OH})$. evidence for a $B_{2,5}$ conformation of the transition state of the enzyme-catalyzed reaction of these families. Previous work with GH47 $\alpha$-mannosidases found that 2 bound in a ${ }^{3} H_{4}$ conformation, implicating a ${ }^{3} S_{1} \rightarrow{ }^{3} H_{4}{ }^{*} \rightarrow{ }^{1} C_{4}$ itinerary for this family (Figure $4 \mathrm{a}$ ). The $B_{2,5}$ conformation has now been observed for mannoimidazole-type inhibitors in complex with $\alpha$ - and $\beta$-mannosidases from five $\mathrm{GH}$ families: $\mathrm{GH} 2,26,38$, 92, and 113 (Figure $4 \mathrm{~b}$ ). A ${ }^{1} S_{5} \leftrightarrow B_{2,5}{ }^{*} \leftrightarrow{ }^{O} S_{2}$ conformational itinerary is common to all of these families, a result that unifies mannosidases which operate through both retaining and inverting, and metal-dependent and metal-independent mechanisms.

Received: September 24, 2013

Published online: December 11, 2013

Keywords: computational chemistry - conformation analysis . enzymes · inhibitors · transition states

[1] G. Z. Lederkremer, Curr. Opin. Struct. Biol. 2009, 19, 515.

[2] L. R. S. Moreira, E. X. F. Filho, Appl. Microbiol. Biotechnol. 2008, 79, 165 .

[3] G. J. Davies, A. Planas, C. Rovira, Acc. Chem. Res. 2012, 45, 308.

[4] S. Fushinobu, V. D. Alves, P. M. Coutinho, Curr. Opin. Struct. Biol. 2013, 23, 652.

[5] D. Crich, Acc. Chem. Res. 2010, 43, 1144.

[6] V. L. Schramm, ACS Chem. Biol. 2013, 8, 71.

[7] T. D. Heightman, A. T. Vasella, Angew. Chem. 1999, 111, 794; Angew. Chem. Int. Ed. 1999, 38, 750.

[8] L. E. Tailford, W. A. Offen, N. Smith, C. Dumon, C. Morland, J. Gratien, M. P. Heck, R. V. Stick, Y. Bleriot, A. Vasella, H. J. Gilbert, G. J. Davies, Nat. Chem. Biol. 2008, 4, 306.

[9] S. Numao, D. A. Kuntz, S. G. Withers, D. R. Rose, J. Biol. Chem. 2003, 278, 48074.

[10] Y. Zhu, M. D. L. Suits, A. J. Thompson, S. Chavan, Z. Dinev, C. Dumon, N. Smith, K. Moreman, Y. Xiang, A. Siriwardena, S. J. Williams, H. J. Gilbert, G. J. Davies, Nat. Chem. Biol. 2010, 6, 125.

[11] A. J. Thompson, J. Dabin, J. Iglesias-Fernandez, A. Ardevol, Z. Dinev, S. J. Williams, O. Bande, A. Siriwardena, C. Moreland, T. C. Hu, D. K. Smith, H. J. Gilbert, C. Rovira, G. J. Davies, Angew. Chem. 2012, 124, 11159; Angew. Chem. Int. Ed. 2012, 51, 10997.

[12] A. Cartmell, E. Topakas, V. M. A. Ducros, M. D. L. Suits, G. J. Davies, H. J. Gilbert, J. Biol. Chem. 2008, 283, 34403.

[13] V. M. A. Ducros, D. L. Zechel, G. N. Murshudov, H. J. Gilbert, L. Szabo, S. Stoll, S. G. Withers, G. J. Davies, Angew. Chem. 2002, 114, 2948; Angew. Chem. Int. Ed. 2002, 41, 2824.

[14] Y. Zhang, J. Ju, H. Peng, F. Gao, C. Zhou, Y. Zeng, Y. Xue, Y. Li, B. Henrissat, G. F. Gao, Y. Ma, J. Biol. Chem. 2008, 283, 31551.

[15] L. E. Tailford, V. M. Ducros, J. E. Flint, S. M. Roberts, C. Morland, D. L. Zechel, N. Smith, M. E. Bjornvad, T. V. Borchert, K. S. Wilson, G. J. Davies, H. J. Gilbert, Biochemistry 2009, 48, 7009.

[16] The FEL of protonated isofagomine is dominated by an intramolecular hydrogen bond which is not relevant to the enzyme-bound species and so the neutral species was studied.

[17] A. J. Thompson, R. J. Williams, Z. Hakki, D. S. Alonzi, T. Wennekes, T. M. Gloster, K. Songsrirote, J. E. Thomas-Oates, T. M. Wrodnigg, J. Spreitz, A. E. Stutz, T. D. Butters, S. J. Williams, G. J. Davies, Proc. Natl. Acad. Sci. USA 2012, 109, 781. [18] See Ref. [8].

[19] D. A. Kuntz, C. A. Tarling, S. G. Withers, D. R. Rose, Biochemistry 2008, 47, 10058. 
[20] See Ref. [10]

[21] W. A. Offen, D. L. Zechel, S. G. Withers, H. J. Gilbert, G. J. Davies, Chem. Commun. 2009, 2484.

[22] L. Petersen, A. Ardevol, C. Rovira, P. J. Reilly, J. Am. Chem. Soc. 2010, 132, 8291.
[23] See Ref. [19].

[24] X. Biarnés, A. Ardèvol, A. Planas, C. Rovira, A. Laio, M. Parrinello, J. Am. Chem. Soc. 2007, 129, 10686.

[25] A. Ardèvol, X. Biarnés, A. Planas, C. Rovira, J. Am. Chem. Soc. 2010, 132,16058 